\title{
Correspondence:
}

\section{Mehran risk score for predicting contrast-induced nephropathy: relevance to Indian population}

With the increasing use of contrast media for both diagnostic and interventional procedures, the development of procedure related contrastinduced nephropathy (CIN) is becoming a subject of concern. CIN is defined as greater than $25 \%$ or $0.5 \mathrm{mg} / \mathrm{dL}$ increase in serum creatinine from baseline within 48 hours after contrast administration. ${ }^{1}$ The reported incidence varies depending on the definition used, the dose and type of contrast administered as well as the presence of risk factors like chronic renal insufficiency, diabetes mellitus, and contrast volume administered. ${ }^{2}$ The cumulative risk of several variables on renal function is thus unknown. ${ }^{3}$

Mehran risk score, ${ }^{3}$ is a simple risk score that could be readily used by clinicians to evaluate individual patient risk to develop CIN after percutaneous coronary interventions (PCI). The risk score for predicting $\mathrm{CIN}$ was calculated according to the following algorithm: hypotension (integer score 5), intra-aortic balloon pump support (integer score 5), congestive heart failure (integer score 5), age greater than 75 years (integer score 4), diabetes mellitus (integer score 3), estimated glomerular function rate (eGFR) less than 60 (integer score 2 to 6 ), pre-existing anaemia (integer score 3 ), and contrast media volume (integer score 1 for each $100 \mathrm{~mL}$ ). The scores less than or equal to $5,6-10,11-16$ and greater than or equal to 16 predicted a CIN risk of $7.5 \%, 14 \%, 26.1 \%$ and $57.3 \%$, respectively. Keeping the utility of the above score, we prospectively applied the same Received: February 08, 2017 to our patient population who underwent PCI in our study.

One hundred and twenty patients scheduled to undergo diagnostic coronary angiography and/ or angioplasty in the Department of Cardiology with baseline serum creatinine less than or equal to $1.2 \mathrm{mg} / \mathrm{dL}$, were recruited. Patients with pre-existing renal disease, hypotension, hyperthyroidism, hypothyroidism, those on glucocorticoid therapy, cardiogenic shock, or allergy to contrast media and patients not willing to participate in the study were excluded. Patients with normal renal function $(\leq 1.2 \mathrm{mg} / \mathrm{dL})$ were recommended liberal intake of oral fluid before the procedure and a record of the input and output was maintained to monitor fluid status. They received lowosmolal contrast agent, iohexol (320mg iodine/ ml, Ominipaque ${ }^{\circledR}$, Jaya Pharmaceutical, Hyderabad, India). Patients with elevated serum creatinine or chronic kidney disease (CKD) received $1 \mathrm{ml} / \mathrm{kg} /$ hour I.V. fluids starting 12 hours prior to and continued 12 hours after the procedure, along with $\mathrm{N}$-acetyl cysteine 600 mg BD, 3 days before and after the procedure and received iso-osmolar contrast medium (IOCM) Iodixonol (VISIPAQUE, from Cipla India).

The baseline and clinical characteristics of the study subjects are shown in Table 1 . The risk score in the study group was $4.2 \pm 30$ which is below 5 and corresponds to $7.5 \%$ risk of CIN. We observed CIN in $27(22.5 \%)$ patients in our study. Further, the score in patients with

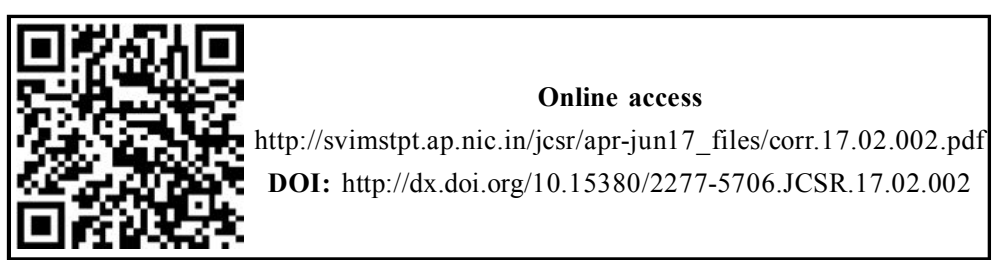


Table 1: Baseline and clinical characteristics in patients with CIN and without CIN Data are presented as are mean \pm SD and number of patients $(\%)$

\begin{tabular}{|c|c|c|c|}
\hline Variables & CIN group $(n=27)$ & Non-CIN group $(n=93)$ & $P$ value \\
\hline Age (years) & $52.81 \pm 8.77$ & $50.28 \pm 8.88$ & 0.194 \\
\hline Male, n (\%) & $26(96.2)$ & $91(97.8)$ & 0.652 \\
\hline $\operatorname{BMI}\left(\mathrm{kg} / \mathrm{m}^{2}\right)$ & $23.79 \pm 3.55$ & $23.81 \pm 2.78$ & 0.970 \\
\hline Diabetes mellitus, n (\%) & $15(55.5)$ & $45(48.3)$ & 0.846 \\
\hline Systolic blood pressure (mm Hg) & $118.14 \pm 12.72$ & $118.46 \pm 12.81$ & 0.911 \\
\hline Diastolic blood pressure (mm Hg) & $76.29 \pm 8.38$ & $76.81 \pm 7.87$ & 0.798 \\
\hline Hypertension, n (\%) & $1(3.70)$ & $7(7.52)$ & 0.473 \\
\hline Smokers, n $(\%)$ & $8(29.6)$ & $48(51.6)$ & 0.052 \\
\hline eGFR by $\mathrm{CG}$ equation, $\mathrm{ml} / \mathrm{min}$ & $97.60 \pm 25.79$ & $89.11 \pm 26.66$ & 0.153 \\
\hline Congestive heart failure, n (\%) & $4(14.8)$ & $16(17.2)$ & 0.771 \\
\hline LVEF $(\%)$ & $51.70 \pm 11.46$ & $51.45 \pm 9.62$ & 0.915 \\
\hline CAG / CAG + PCI, n (\%) & $12(44.4) / 15(55.5)$ & $37(39.7) / 56(60.2)$ & 0.667 \\
\hline Volume of contrast $(\mathrm{ml})$ & $62.8 \pm 28.8$ & $60.4 \pm 24.1$ & 0.732 \\
\hline Angiographic characteristic, $\mathrm{n}(\%)$ & & & 0.307 \\
\hline One vessel & $9(33.3)$ & $43(46.23)$ & \\
\hline Two vessels & $8(29.62)$ & $30(32.25)$ & \\
\hline Multi-vessels & $6(22.22)$ & $9(9.67)$ & \\
\hline \multicolumn{4}{|l|}{ Drugs } \\
\hline ACE inhibitor, n (\%) & - & $2(2.15)$ & 1.000 \\
\hline Diuretics, $\mathrm{n}(\%)$ & $10(37.0)$ & $2(2.15)$ & 1.000 \\
\hline$\beta$ - blockers, n (\%) & $8(29.6)$ & $5(5.37)$ & 0.165 \\
\hline Aspirin, $n(\%)$ & $23(85.1)$ & $85(91.3)$ & 0.464 \\
\hline Statins, n $(\%)$ & $11(40.7)$ & $18(19.3)$ & 0.039 \\
\hline Mehran risk score & $4.33 \pm 0.60$ & $4.10 \pm 0.39$ & 0.620 \\
\hline
\end{tabular}

$\mathrm{BMI}=$ body mass index, $\mathrm{CIN}=$ contrast-induced nephropathy, eGFR = estimated glomerular filtration rate, $\mathrm{CG}$ equation= Cockcroft \& Gault equation, $\mathrm{LVEF}=$ left ventricular ejection fraction, $\mathrm{CAG}=$ coronary angiography, $\mathrm{PCI}=$ percutaneous coronary intervention, $\mathrm{ACE}=$ angiotensin-onverting enzyme.

diabetes mellitus who developed CIN ( $\mathrm{n}=15$, $25 \%$ ) was higher than patients without diabetes mellitus ( $\mathrm{n}=12,20 \%)(6.2 \pm 2.8$ vs $1.9 \pm 2.0$; $\mathrm{p}<0.001)$ since presence of diabetes itself carries an integer score of 3 . A risk score between 6-10 corresponds to a risk of CIN of $14 \%$ and the observed incidence in both the diabetic $(25 \%)$ and nondiabetic subgroups is higher $(20 \%)$ than that expected for their corresponding risk scores (14\% and $7.5 \%$ respectively).

Incidence of CIN in our study is much higher than that expected in patients with normal baseline renal function and for the corresponding Mehran risk score. ${ }^{3}$ The incidence of CIN depends on the type of contrast medium administered. Low-osmolal, non-ionic contrast media have been shown to be more nephrotoxic compared to iso-osmolal contrast medium. ${ }^{4}$ Iohexol, used in the present study has been shown to be associated with increased incidence of CIN. ${ }^{5}$ The type of prophylaxis administered prior to the procedure also influences the outcome. Table 2 gives a summary of Indian studies on the effect of prophylactic measures on the development of CIN. Patients with normal baseline renal function receiving $\mathrm{N}$-acetyl-cysteine, ${ }^{6}$ allopurinol, ${ }^{6}$ theophylline ${ }^{7}$ or dopamine ${ }^{8}$ have been shown to have a lesser incidence of CIN (Table 2). Hydration was performed with $1 \mathrm{~mL} /$ $\mathrm{kg} / \mathrm{h}$ of half-normal saline for $4-12 \mathrm{~h}$ before PCI and $18-24 \mathrm{~h}$ after PCI in the study by Mehran et al, ${ }^{3}$ as against unrestricted use of oral fluids in the present study. This shows that preventive strategies being followed in patients with normal baseline renal function should be given importance as is being done for those with impaired renal function. The use of statins was significantly $(p=0.039)$ more in the CIN 


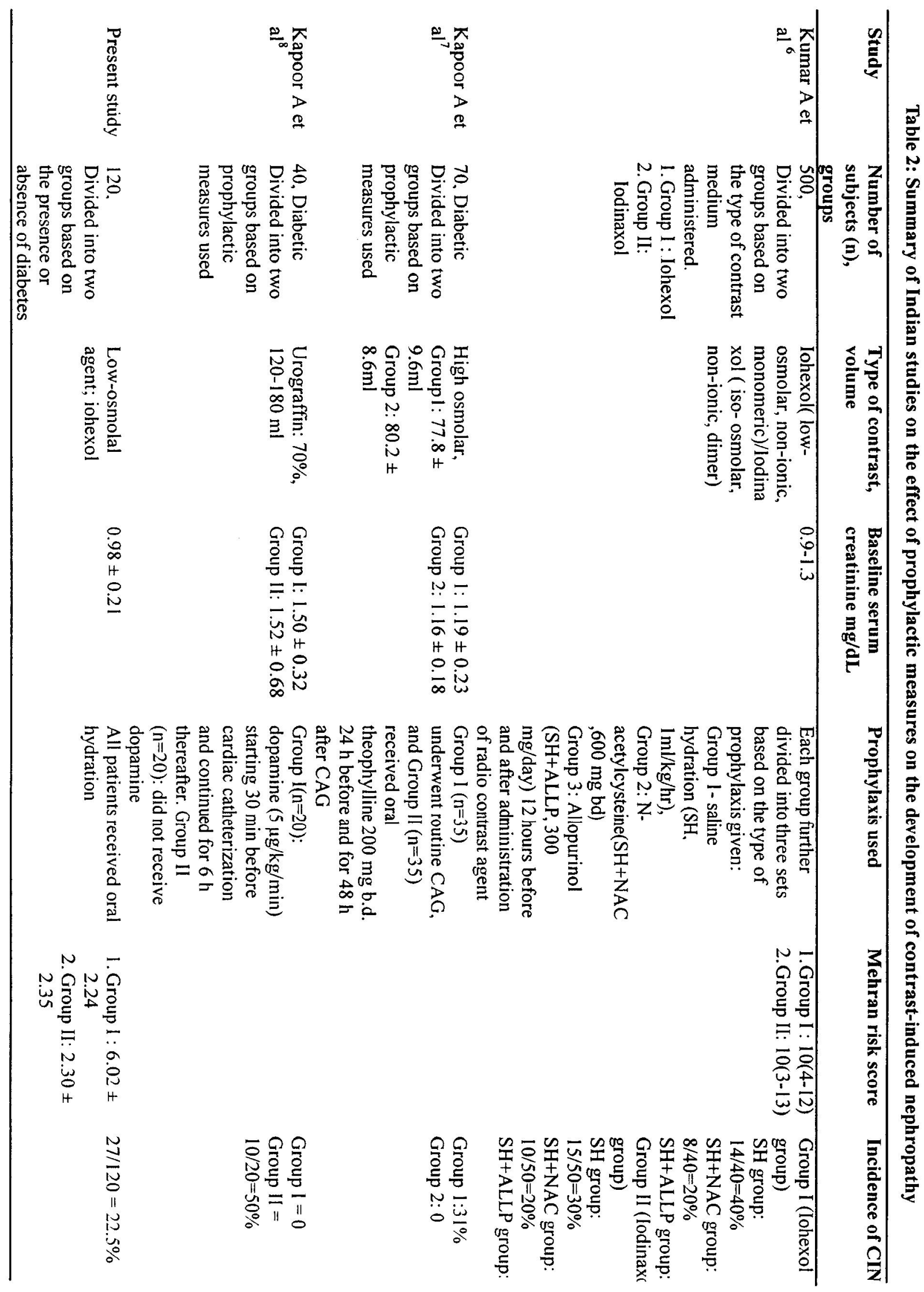


group compared to the non-CIN group. Contrary to our finding, literature states that statins have a beneficial effect on reducing CIN incidence in patients with baseline renal impairement. ${ }^{9}$ Similarly, another study ${ }^{10}$ showed that statin pretreatment significantly decreased the risk of CIN in CAD patients undergoing PCI. However, a study ${ }^{11}$ which classified patients based on Mehran score for risk development of CIN and analysed the effect of statins observed a higher incidence of CIN in patients on statins as compared with patients not on statins. However, this difference was not observed after propensity-based adjustment for reciept of statins. Hence, this needs further probing.

In a study from India, ${ }^{6}$ the Mehran risk score ${ }^{3}$ of the patients ranged from 3-10 and observed incidence was $40 \%$ in the group that received iohexol as the contrast medium and normal saline hydration prior to the procedure. The baseline renal function in their subjects was also normal (serum creatinine $0.9-1.3 \mathrm{mg} / \mathrm{dL}$ ). Similarly, our study shows that the incidence of CIN is higher than that predicted by Mehran risk score. ${ }^{3}$ Also, the risk score did not differ between the CIN and non-CIN groups implying that it is unable to predict CIN in our population. Hence, there is a need to further validate this widely acceptable risk scoring system in a larger number of patient population for its application to assess the risk of CIN in Indian population. Also, a new risk scoring system which is easy to implement in clinical practice needs to be developed especially for those with normal baseline renal function who are often neglected assuming them to be at low risk.

\section{REFERENCES}

1. Morcos SK, Thomsen HS, Webb JA. Contrastmedia-induced nephrotoxicity: a consensus report.
Contrast Media Safety Committee, European Society of Urogenital Radiology (ESUR) Eur Radiol 1999;9:1602-13.

2. Parfrey PS, Griffiths SM, Barrett BJ, Paul MD, Genge M, Withers J, et al. Contrast materialinduced renal failure in patients with diabetes mellitus, renal insufficiency, or both. A prospective controlled study. N Engl J Med 1989;320:143-53.

3. Mehran R, Aymong ED, Nikolsky E, Lasic Z, Iakovou I, Fahy M, et al. A simple risk score for prediction of contrast-induced nephropathy after percutaneous coronary intervention: Development and initial validation. J Am Coll Cardiol 2004;44:1393-9.

4. Aspelin P, Aubry P, Fransson SG, Strasser R, Willenbrock R, Berg KJ; Nephrotoxicity in HighRisk Patients Study of Iso-Osmolar and LowOsmolar Non-Ionic Contrast Media Study Investigators. Nephrotoxic effects in high-risk patients undergoing angiography. N Engl J Med 2003;348:491-9.

5. Solomon R, Dumouchel W. Contrast media and nephropathy: Findings from systematic analysis and Food and Drug Administration reports of adverse effects. Invest Radiol 2006;41:651-60.

6. Kumar A, Bhawani G, Kumari N, Murthy KS , Lalwani V, Raju ChN . Comparative study of renal protective effects of allopurinol and Nacetylcysteine on contrast induced nephropathy in patients undergoing cardiac catheterization. $\mathrm{J}$ Clin Diagn Res 2014 8:HC03-HC07.

7. Kapoor A, Kumar S, Gulati S, Gambhir S, Sethi RS, Sinha N. The role of theophylline in contrastinduced nephropathy: a case-control study. Nephrol Dial Transplant 2002; 17:1936-41.

8. Kapoor A, Sinha N, Sharma RK, Shrivastava S, Radhakrishnan S, Goel PK, Bajaj R. Use of dopamine in prevention of contrast induced acute renal failure a randomised study. Int J Cardiol 1996 53:233-6.

9. Singh N, Lee JZ, Huang JJ, Low SW, Howe C, Pandit A, et al. Benefit of statin pretreatment in prevention of contrast-induced nephropathy in different adult patient population: systematic review and meta-analysis. Open Heart 2014;1:e000127. 
10. Hoshi T, Sato A, Kakefuda y, Hanunari T, Watabe H, Ojima E et.al. Preventive effect of statin pre treatment on contrast-induced acute kidney injury in patients undergoing coronary angioplasty: propensity score analysis from a multicenter registry. Int J Cardiol 2014;171:243-9.
11. Kandula P, Shah R, Singh N, Markwell SJ, Bhensdadia N, Navaneethan SD. Statins for prevention of contrast-induced nephropathy in patients undergoing non-emergent percutaneous coronary intervention. Nephrology (Carlton). 2010;15:165-70.

Aparna R.Bitla, ${ }^{1}$

P. Sandeep, ${ }^{2}$

V.L. Ashalatha, ${ }^{1}$

A.Y.Lakshmi, ${ }^{3}$

P.V.L.N. Srinivasa Rao, ${ }^{1}$

D. Rajasekhar, ${ }^{4}$

V. Siva Kumar ${ }^{5}$

Departments of ${ }^{1}$ Biochemistry, ${ }^{3}$ Radiology, ${ }^{4}$ Cardiology, ${ }^{5}$ Nephrology, Sri Venkateswara Institute of Medical Sciences,

Tirupati and Department of ${ }^{2}$ Nephrology,

Chalmeda Anand Rao Institute of Medical Sciences, Bommakal

Received: February 08, 2017; Revised manuscript received: February 22, 2017; Accepted: March 03, 2017

Bitla AR, Sandeep P, Ashalatha VL, Lakshmi AY, Srinivasa Rao PVLN, Rajasekhar D, Siva Kumar V. Mehran risk score for predicting contrast-induced nephropathy: Relevance to Indian population. J Clin Sci Res 2017;6:133-37. DOI: http:// dx.doi.org/10.15380/2277-5706.JCSR.17.02.002. 